“ (C) 2012 IEEE. Personal use of this material is permitted. Permission from IEEE must be obtained for all other uses, in any current or future media, including

reprinting/republishing this material for advertising or promotional purposes, creating new collective works, for resale or redistribution to servers or lists, or reuse of any copyrighted component of this work in other works." 


\title{
A Pattern Reconfigurable U-Slot Antenna and Its Applications in MIMO Systems
}

\author{
Pei-Yuan Qin, Y. Jay Guo, Senior Member, IEEE, Andrew R. Weily, Member, IEEE, \\ and Chang-Hong Liang, Senior Member, IEEE
}

\begin{abstract}
A new compact pattern reconfigurable U-slot antenna is presented. The antenna consists of a $U$-slot patch and eight shorting posts. Each edge of the square patch is connected to two shorting posts via PIN diodes. By switching between the different states of the PIN diodes, the proposed antenna can operate in either monopolar patch or normal patch mode in similar frequency ranges. Therefore, its radiation pattern can be switched between conical and boresight patterns electrically. In addition, the plane with the maximum power level of the conical pattern can be changed between two orthogonal planes. Owing to a novel design of the switch geometry, the antenna does not need dc bias lines. The measured overlapping impedance bandwidth $\left(\left|S_{11}\right|<-10\right.$ dB) of the two modes is $6.6 \%$ with a center frequency of $5.32 \mathrm{GHz}$. The measured radiation patterns agree well with simulated results. The antennas are incorporated in a $2 \times 2$ multiple-input-multiple-output (MIMO) orthogonal frequency division multiplexing (OFDM) system to demonstrate the improvement in system capacity. In the real-time MIMO-OFDM channel measurement, it is shown that compared to omnidirectional antennas, the pattern reconfigurable antennas can enhance the system capacity, with $17 \%$ improvement in a line-of-sight (LOS) scenario and $12 \%$ in a non-LOS (NLOS) scenario at a signal-to-noise ratio (SNR) of $10 \mathrm{~dB}$.
\end{abstract}

Index Terms-Microstrip antennas, reconfigurable antennas, multiple-input-multiple-output (MIMO), slot antennas.

\section{INTRODUCTION}

$\mathbf{I}_{\mathrm{s} i}^{\mathrm{n}}$ $\mathrm{n}$ the past few years, reconfigurable antennas have received significant attention due to their ability to improve the performance of wireless communication systems [1]-[10]. Typical parameters of an antenna that can be reconfigured are frequency, radiation pattern, polarization or combinations of the above. Pattern reconfigurable antennas have the potential to avoid noise sources by changing the null position, to save energy by better directing the signal toward intended users and

Manuscript received, June 29, 2010; revised March 2 and May 9, 2011. This work was supported by the DIISR Australia-China special fund CH080270.

P.-Y. Qin is with the Science and Technology on Antenna and Microwave Laboratory, Xidian University, Xi'an, Shaanxi 710071, China, with the Department of Electronic Engineering, Macquarie University, North Ryde, NSW 2109, Australia, and the CSIRO ICT Centre, Epping, NSW 1710 Australia. (e-mail:Peiyuan.qin@csiro.au).

Y. J. Guo and A. R. Weily are with the CSIRO ICT Centre, Epping, NSW 1710, Australia.

C.-H. Liang is with the Science and Technology on Antenna and Microwave Laboratory, Xidian University, Xi'an, Shaanxi, 710071, China. to provide larger coverage by redirecting the main beam [7]-[10]. Among the reported pattern reconfigurable antenna designs, several [8]-[10] feature the capability to switch between boresight and conical patterns. A conical radiation pattern is generally one for which the maximum directivity is off boresight (where boresight corresponds to the direction normal to the plane containing the antenna) and the pattern shape resembles a cone. In [8], a wide-band L-probe circular patch antenna with dual feeds was presented. Unfortunately, an integrated matching network consisting of switches needs to be designed in order to reconfigure the radiation pattern electrically. In [9] and [10], single feed pattern reconfigurable square-ring patch antennas were designed with air gaps to increase the impedance bandwidth, and dc bias networks were used to drive the PIN diodes. In most conventional reconfigurable antenna structures, dc bias lines are required to control the switching elements, which can make the whole antenna structure more complicated or even degrade the antenna performance. This hinders the wide applications of reconfigurable antennas. Therefore, a reconfigurable antenna structure without bias lines is desired.

Very recently, reconfigurable antennas have found new applications in adaptive multiple-input-multiple-output (MIMO) systems [11]-[20], enabling the dynamic change of radiating characteristics of each antenna element according to the usually fast changing channel conditions. Generally, there are two methods to increase the MIMO system capacity by employing reconfigurable antennas. The first is to reduce the correlation of sub-channels by using polarization or pattern reconfigurable antennas. Specifically, this is implemented by switching between different configurations of reconfigurable antenna arrays according to the varying channel conditions. The polarization or pattern diversity in some of the configurations can be used to realize low correlation of the sub-channels. The second is to increase the signal power received by switching the antenna radiation patterns according to the channel information. In [12]-[15], the capacity of a MIMO system was improved by using polarization reconfigurable antennas to reduce the sub-channel correlation. In [16]-[20], pattern reconfigurable antennas were incorporated in MIMO systems to enhance the system capacity. In [16], a MIMO system with pattern 
reconfigurable antennas was tested in an anechoic chamber with artificial objects acting as the scatterers that made up a multipath environment. The system capacity increase was mostly attributed to the increase of average receiver signal-to-noise ratio (SNR) by changing the main beam direction. In [17]-[20], the capacity of a MIMO system was increased by exploiting antenna pattern diversity to introduce sub-channel decorrelation to the MIMO system. For the experiments in [17], the reference antenna was set to be one configuration of the reconfigurable antenna. It is known that an omnidirectional antenna can receive rich multipath, which can lead to low sub-channel correlation [20]. Therefore, a capacity comparison between systems with reconfigurable and omnidirectional antennas is necessary for highlighting the effect of pattern diversity on the sub-channel correlation.

In this paper, a new pattern reconfigurable microstrip U-slot patch antenna using eight PIN diodes is proposed. Eight shorting posts are implemented around the patch to change the operating mode of the antenna from monopolar patch mode to normal patch mode. In addition, the two modes are designed to resonate in similar frequency ranges. Therefore, the proposed antenna can electrically reconfigure the radiation pattern between conical and boresight patterns with an overlapping impedance bandwidth. Furthermore, the plane with the maximum power level of the conical pattern can be varied between two orthogonal planes when the antenna operates in the monopolar patch mode.

To demonstrate the benefit of using the proposed reconfigurable antennas to increase the system capacity, four antennas have been employed in a $2 \times 2$ MIMO-OFDM demonstrator. Omnidirectional antennas are used as a reference for capacity comparison. Channel measurements conducted in both line-of-sight (LOS) and non-LOS (NLOS) indoor environments show significant capacity enhancement.

Part of this work has been described in [21]. The present paper extends the work in [21] significantly by providing the antenna design principles and showing the equivalent circuits of the antenna with PIN diodes. We also present a parametric study on the antenna resonant frequency and describe the measured reflection coefficient, radiation pattern, gain and efficiency of the antenna. In addition, a discussion on the measured realized gain of the antenna is given. Furthermore, the pattern reconfigurable antenna is applied to a MIMO- orthogonal frequency division multiplexing (OFDM) system and the capacity comparison between systems with pattern reconfigurable antennas and omnidirectional antennas is shown.

Compared with antennas in [8]-[10], the proposed antenna has three main advantages. Firstly, only a single bias-tee, which superimposes the bias voltage on the RF signal, is needed to control the PIN diodes in the proposed antenna. Consequently, the complex bias network for PIN diodes or the matching network for dual feeds is not required as part of the printed antenna structure, which greatly simplifies the device. Secondly, the proposed antenna is compact and of low profile since it is designed on a single layer microwave substrate.

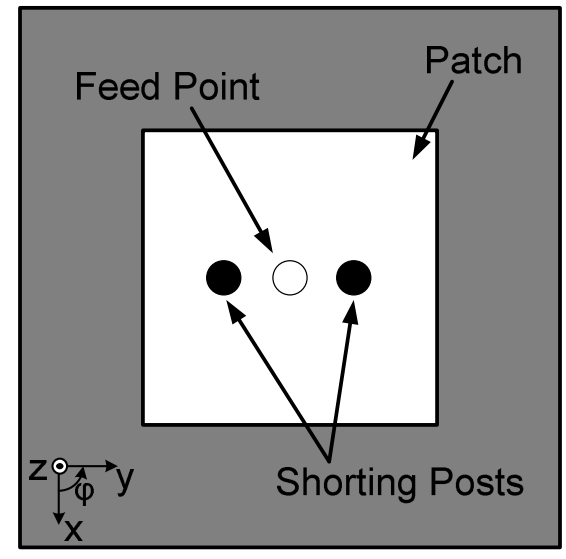

(a)

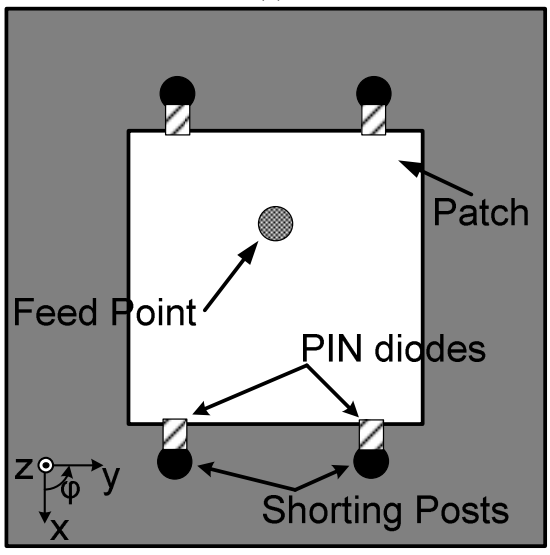

(b)

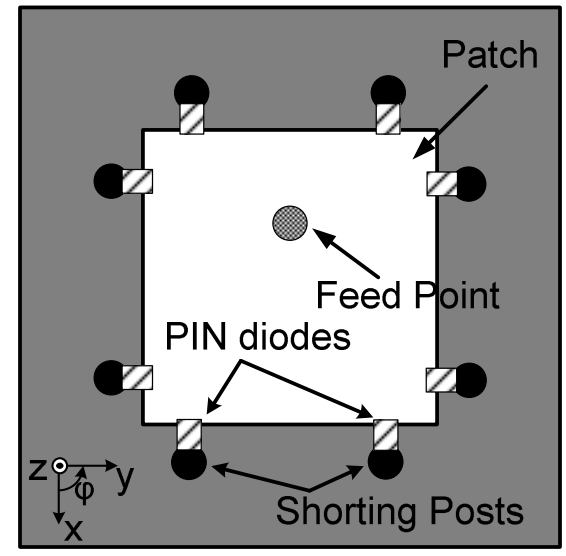

(c)

Fig. 1 Configurations of the patch antenna with PIN diodes (a) antenna in Ref. [23] with two shorting posts; (b) antenna with four shorting posts; (c) antenna with eight shorting posts.

Thirdly, compared to antennas in [8]-[10] which can switch between two radiation patterns, the proposed antenna has three different patterns in a similar frequency band. The larger number of patterns gives the proposed antenna more flexibility to improve the system capacity of a wireless link. However, a disadvantage of the proposed antenna is the use of eight PIN diodes, the loss of which will reduce the antenna realized gain. The effect of the loss of PIN diodes on the antenna gain is discussed at the end of Section IV.

This paper is organized as follows. In Section II, the operating principle and structure of the proposed antenna are 


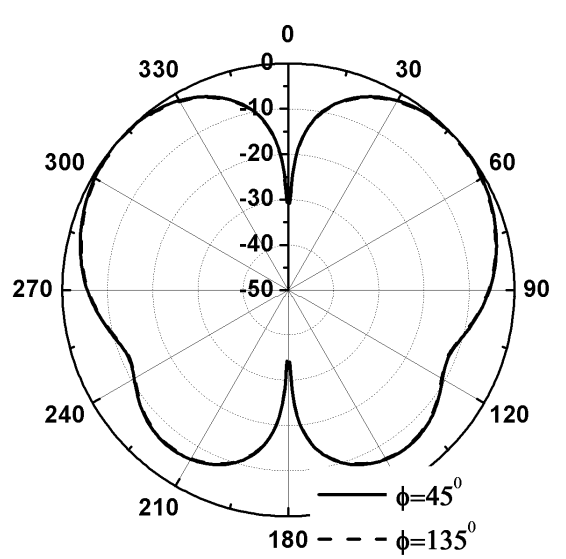

(a)

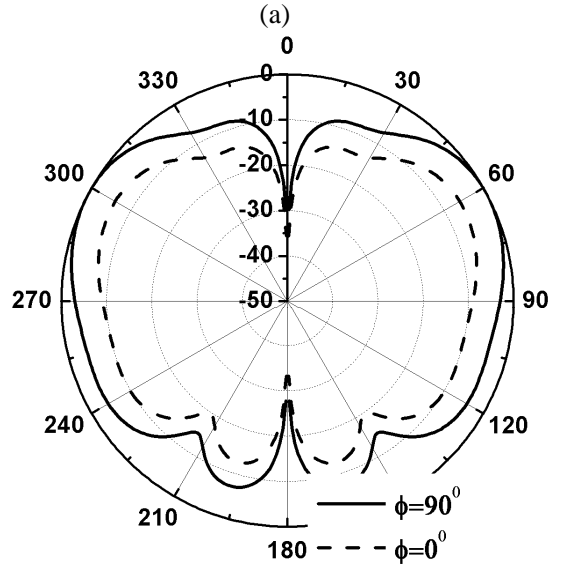

(b)

Fig. 2 Simulated normalized radiation pattern (a) antenna in Ref. [23]; (b) antenna in Fig. 1 (b).

described. Section III presents parametric studies of the antenna. Simulated and measured performances of the antenna are provided in Section IV. In Section V, the effects of the pattern reconfigurable antenna on the capacity of a $2 \times 2$ MIMO-OFDM system in indoor environments are analyzed. The paper concludes in Section VI with a summary and suggestions for further work.

\section{ANTENNA DESIGN}

\section{A. Design Guidelines}

Microstrip patch antennas excited in the normal patch mode for boresight radiation and monopolar mode for conical radiation have been reported in [22] and [23], respectively. In [23], the monopolar mode is excited by two shorting posts located to the left and right of the feeding point, which is shown in Fig. 1(a). If PIN diodes are used to connect the shorting posts and the microstrip patch, it is possible for this single antenna to operate in monopolar patch mode or normal patch mode by switching between the different states of the PIN diodes. However, according to [23], the resonant frequency of the monopolar mode is roughly a factor of 2.5 below the fundamental normal patch mode. In order to design an antenna with two modes resonating at similar frequency ranges and having a reasonably wide frequency bandwidth, we have taken three measures in the design process.
Firstly, compared with the antenna in [23], we use two shorting posts at either side of the feed point. In order to simplify the bias network, the shorting posts are implemented around the edge of the patch and connected to the patch via PIN diodes, which is shown in Fig. 1(b). It is well known that the resonant frequency of a patch antenna loaded with reactive components can be varied depending on the type of reactance used [24], [25]. When the shorting posts are connected to the microstrip patch, the antenna operates in the monopolar patch mode. In this case, the increase in the number of shorting posts will increase the resonate frequency. On the other hand, when all the shorting posts are disconnected from the patch, the antenna operates in the normal patch mode. In this case, the increase in the number of shoring posts will reduce the resonant frequency. Therefore, the frequency difference between the two modes becomes smaller as the number of the shorting posts increases. The effect of the number of shorting posts on the antenna resonant frequency will be detailed in Section III.

Secondly, we examine the normalized far-field radiation patterns for the antennas operating in monopolar patch mode in Fig. 1 (a) and (b), which is given in Fig. 2 (a) and (b), respectively. The antennas are analyzed using the time domain solver of CST Microwave Studio [26]. For the antenna in Fig. 1 (a), as is described in [23], two identical conical patterns are located in two orthogonal planes $\varphi= \pm 45^{\circ}$. For the antenna in Fig. 1 (b), the maximum power level of the conical pattern in the plane $\varphi=90^{\circ}$ are $6 \mathrm{~dB}$ greater than that in the plane $\varphi=0^{\circ}$. The qualitative explanation of this behavior is that the two shorting posts connected to each edge of the patch can be treated as a shorting wall that suppresses the E-field at the centre of that edge. Therefore, the maximum power level in the plane $\varphi=0^{\circ}$ is lower than that in the plane $\varphi=90^{\circ}$. In order to have another similar conical pattern with the maximum power level located at the plane $\varphi=0^{\circ}$ for the antenna in Fig. 1 (b), four shorting posts are inserted into the substrate around the other two edges of the microstrip patch, which can be seen in Fig. 1 (c).

Finally, as the probe-fed microstrip patch antenna has a narrow impedance bandwidth that precludes its use in typical communication systems, a U-slot is etched on the patch to increase its impedance bandwidth [27].

\section{B. Antenna Structure}

The layout of the proposed pattern reconfigurable U-slot antenna is shown in Fig. 3. A U-slot is inserted into a square patch of dimensions $L 1 \times L 1$. Each side of the patch is connected with two shorting posts via PIN diodes. The radius of the shorting posts $r$ is $0.7 \mathrm{~mm}$. The feeding probe connected to the U-slot patch through the ground plane and substrate is offset from the top edge of the patch by $L 5$. Since the length of the PIN diode is less than the width of the gap $L 8$, conducting ring pads are placed around the shorting posts to enable attachment of the PIN diodes. The parameters and dimensions of the antenna are given in Table I.

Beam lead PIN diodes (MA4AGBLP912) are used as the switching elements. The equivalent circuit used in the simulation software is presented in Fig. 4. According to the PIN 


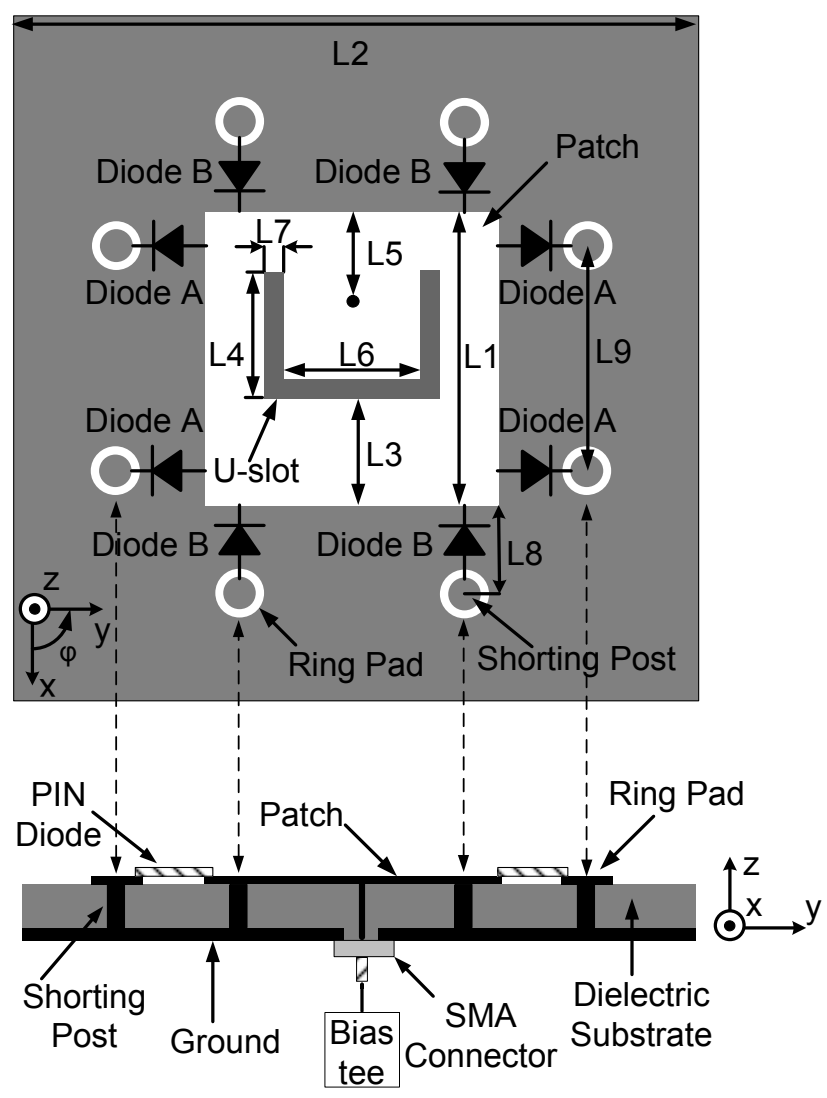

Fig. 3 Schematics of the pattern reconfigurable U-slot antenna.

Table I Dimensions of the pattern reconfigurable U-slot antenna

\begin{tabular}{|c|c|c|c|c|c|}
\hline Parameters & L1 & L2 & L3 & L4 & L5 \\
\hline Value (mm) & 12.6 & 50 & 5.5 & 5.4 & 4.2 \\
\hline Parameters & L6 & L7 & L8 & L9 & r \\
\hline Value (mm) & 4.6 & 0.7 & 1.7 & 9.8 & 0.7 \\
\hline
\end{tabular}

diode datasheet [28], the resistor $\left(R_{S}\right)$ is $4 \Omega$ in the forward bias state and the capacitor $\left(C_{T}\right)$ in the parallel circuit is $0.025 \mathrm{pF}$ in the reverse bias state. The resistor $\left(R_{P}\right)$ is $10 \mathrm{k} \Omega$ representing the net dissipative resistance of the diode in the reverse bias state. For the zero bias state, the value of $R_{P}$ is almost infinity which is equal to an open circuit and the loss caused by the resistor $\left(R_{P}\right)$ is negligible.

The orientation of the diodes is also shown in Fig. 3. As all PIN diodes are mounted across the ground and the center patch, only a bias tee attached to the SMA connector is needed to control the PIN diodes. When the bias voltage is supplied from the coaxial probe, opposite bias conditions are applied to diodes in group A and B due to their reversed orientation. When the dc voltage is zero, all diodes are turned off. In this case, the antenna operates in the normal patch mode and radiates a boresight pattern (State I). When the dc voltage is negative, diodes in group B are on, and the other diodes are off. In this case, the antenna has four shorting posts connected and can be regarded as a monopolar patch antenna, which radiates a conical pattern

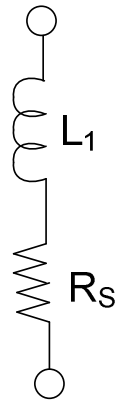

(a)

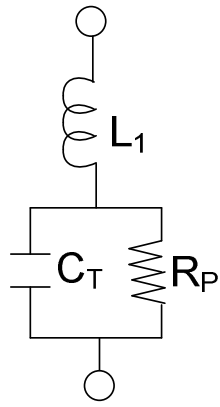

(b)
Fig. 4 Equivalent circuit for PIN diode (a) forward bias (b) reverse bias.

Table II Three states of the pattern reconfigurable U-slot antenna

\begin{tabular}{|c|c|c|c|}
\hline & Diodes Group A & Diodes Group B & $\begin{array}{c}\text { Radiation } \\
\text { Patterns }\end{array}$ \\
\hline State I & Zero Bias & Zero Bias & $\begin{array}{c}\text { Boresight } \\
\text { pattern }\end{array}$ \\
\hline State II & Reverse Bias & Forward Bias & $\begin{array}{c}\text { Conical pattern } \\
\text { with maximum } \\
\text { power level in } \\
\text { the z-y plane }\end{array}$ \\
\hline State III & Forward Bias & Reverse Bias & $\begin{array}{c}\text { Conical pattern } \\
\text { with maximum } \\
\text { power level in } \\
\text { the z-x plane }\end{array}$ \\
\hline
\end{tabular}

with the maximum power level in the z-y plane (State II). Changing the polarity of the dc voltage from negative to positive, diodes in group A are on, and all the other diodes are off. In this case, a similar conical pattern can be observed with the maximum power level in the $\mathrm{z}$-x plane (State III). The possible radiation patterns of the reconfigurable $\mathrm{U}$-slot antenna and the corresponding diode states are summarized in Table II.

\section{PARAMETRIC STUDY}

Three important parameters which affect the input reflection coefficient of the two modes of the proposed reconfigurable antenna are the radius of the shorting posts, the distance between shorting posts and the patch $L 8$, and the number of the shorting posts. Also it is well known that the U-slot plays an important role on the performance of the patch antenna. Since a parametric study of the U-slot has already been reported [29], we only examine the effects of the former three parameters in this paper. The other parameters remain constant and their values are given in Table I. As the shorting posts have the same effect on the reflection coefficient of States II and III for the monopolar patch mode, only the results for State II are presented for the parametric analysis.

In order to better analyze the effect of the parameters, the equivalent circuits of the antenna with PIN diodes for States I and II are given in Fig. 5 (a) and (b), respectively. In the equivalent circuits, a parallel resonant $R L C$ circuit is used to model the patch antenna with a U-slot. The purpose of the electrical models in Fig. 5 is to give a physical insight into the behavior of the antenna for the parametric analysis, but not to exactly predict the antenna input impedance. A similar method has been used in [30] for the parametric analysis of a microstrip patch antenna. 


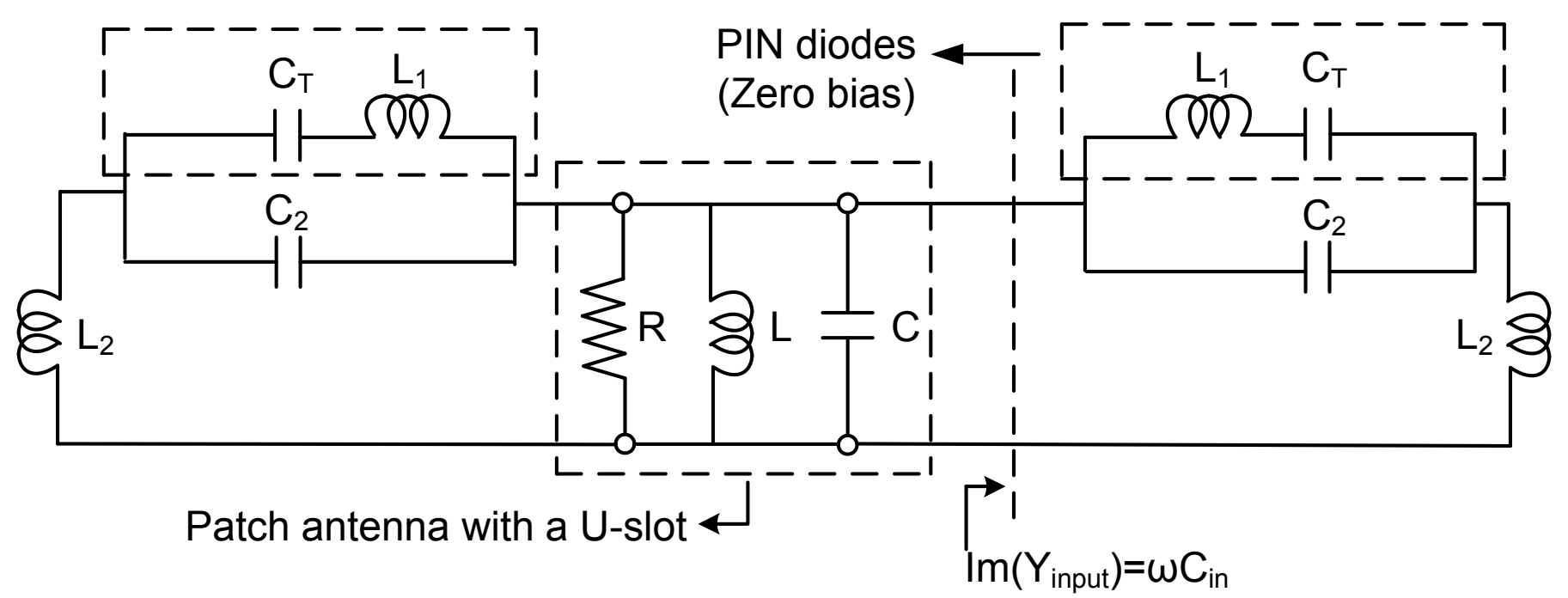

(a)

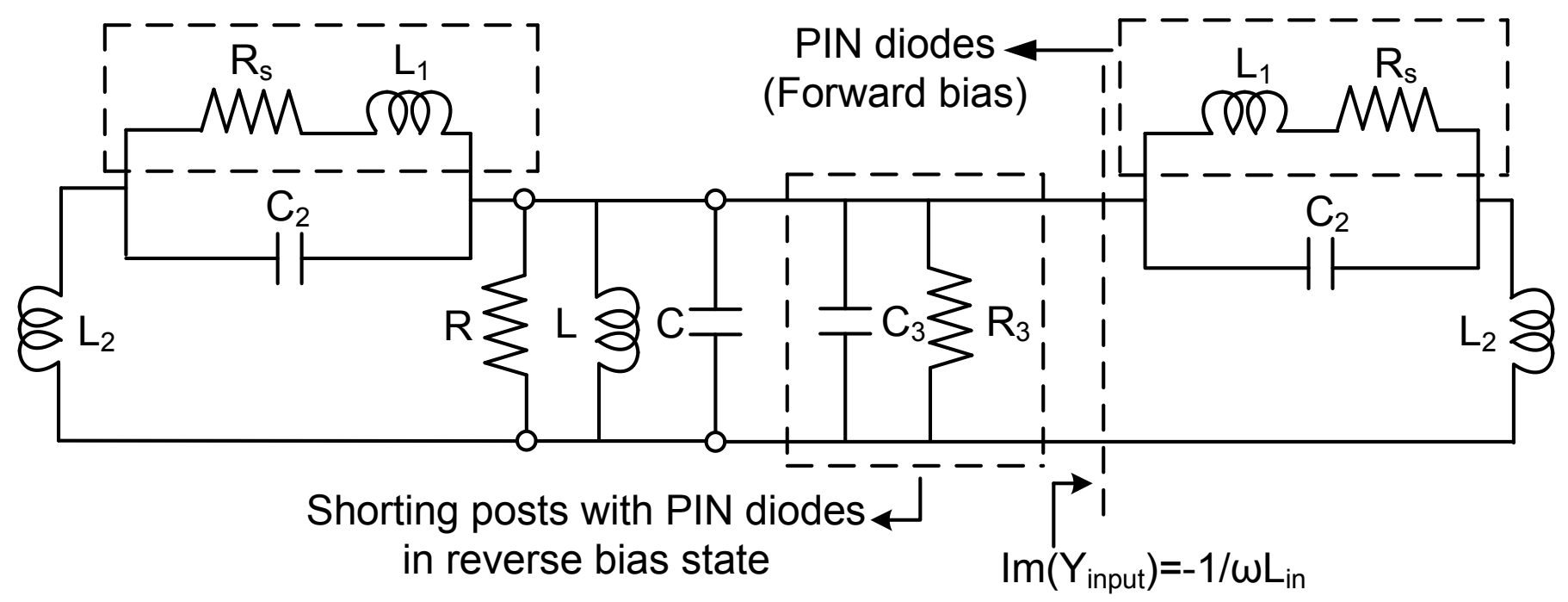

(b)

Fig. 5 The equivalent circuits of the proposed antenna for (a) State I (normal patch mode) and (b) State II (monopolar patch mode).

Fig. 5(a) shows the equivalent circuit of the antenna operating in the normal patch mode. The microstrip patch with a U-slot is represented by a $R L C$ circuit $(R, L, C)$. For the normal patch mode, only the PIN diodes and the shorting posts that are attached to the radiating edge are considered in the equivalent circuit, and the effects of the two PIN diodes and shorting posts at each edge are combined together to simplify the equivalent circuit. As the PIN diodes are all zero bias, according to the PIN diode datasheet, $R_{P}$ is almost infinity. Therefore, the equivalent circuit of the PIN diode in this state is an inductor $L_{l}$ in series with a capacitor $C_{T}$. The parasitic capacitance between the shorting posts and the patch is modeled by a capacitor $C_{2}$. The shorting post is represented by a shunt inductor $L_{2}$. The imaginary part of the input admittance $Y_{\text {in }}$ from the reference plane on the right hand side of Fig. 5 (a) is investigated by using CST Microwave Studio. Simulation results show that it is capacitive with a capacitance $C_{i n}$ within the antenna operating frequency range.
Fig. 5 (b) shows the equivalent circuit of the antenna operating in the monopolar patch mode. As with the circuit of the normal patch mode, the microstrip patch with a U-slot is also represented by a $R L C$ circuit $(R, L, C)$. In the monopolar patch mode (State II), four PIN diodes (diodes A) are reverse bias. According to the analysis in the last paragraph, the total effect of the shorting posts with PIN diodes in zero bias state is capacitive. The only difference between the zero and reverse bias states is the value of the net dissipative resistor $R_{P}$ of the PIN diodes. Therefore, a capacitor $C_{3}$ is used to model the total capacitive effect of the shorting posts connected with diodes A. A resistor $R_{3}$ is used to represent the losses in the four PIN diodes. On the other hand, four shorting posts are connected to the patch by PIN diodes B that are in the forward bias state. Those PIN diodes are modeled by an inductor $L_{l}$ in series with a resistor $R_{s}$. In addition, the effects of the shorting posts and the parasitic capacitance between the patch and the shorting posts are represented by an inductor $L_{2}$ and a capacitor $C_{2}$, 


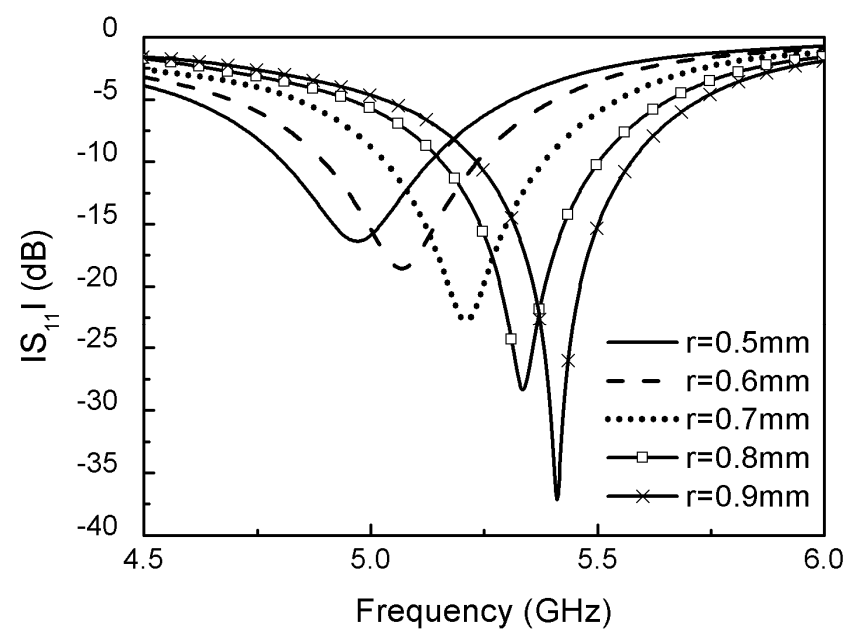

(a)

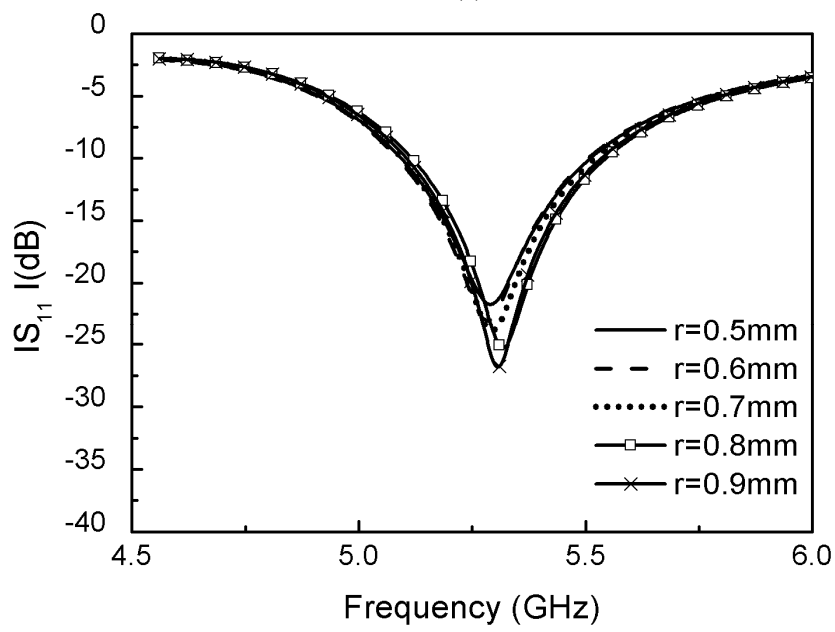

(b)

Fig. 6 Simulated performance of the proposed antenna as a function of $r$ (a) input reflection coefficient of monopolar patch mode; (b) input reflection coefficient of normal patch mode.

respectively. The imaginary part of the input admittance $Y_{\text {in }}$ from the reference plane on the right hand side of Fig. 5 (b) is investigated. Simulation results show that it is inductive with an inductance $L_{i n}$ within the antenna operating frequency range.

\section{A. The radius of shorting posts $r$}

Fig. 6 (a) and (b) show the effects of the radius of shorting posts $r$ on the resonant frequencies of the monopolar patch and normal patch modes, respectively. It is observed that the resonant frequency of the monopolar patch mode increases with the radius of the shorting posts. This is due to the fact that when the radius increases the inductance from the shorting posts reduces, which makes $L_{i n}$ decrease; hence, the resonant frequency of the parallel circuit in Fig. 5 (b) increase. For the normal patch mode, the resonant frequency remains almost unaffected by the radius change of shorting posts. This is because that $C_{i n}$ in Fig. 5 (a) is almost stable within the changing range of the shorting post radius, which is evidenced by the simulation results. Therefore, the radius of the shorting posts can change the resonant frequency of the monopolar patch mode but has little effect on that of the normal patch mode.

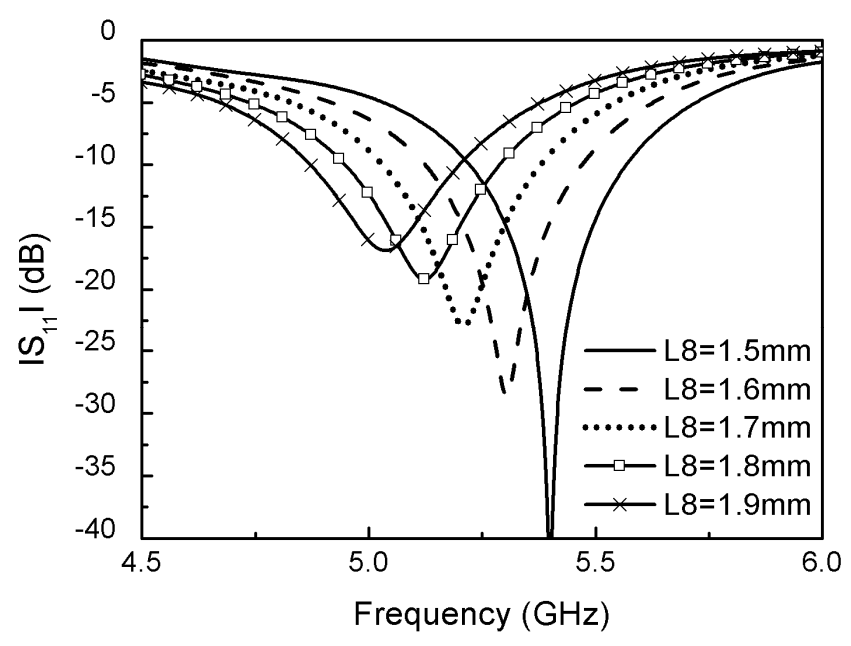

(a)

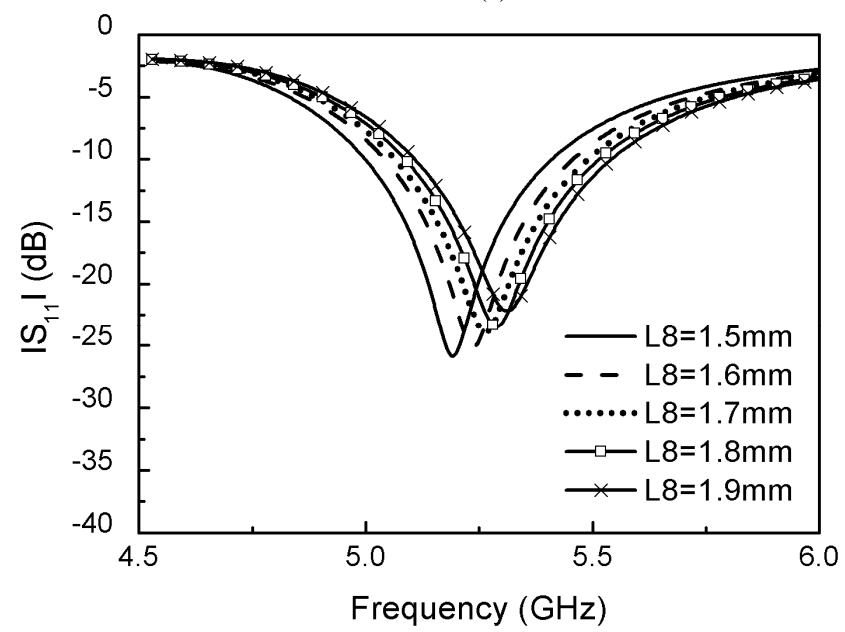

(b)

Fig. 7 Simulated performance of the proposed antenna as a function of $L 8$ (a) input reflection coefficient of monopolar patch mode; (b) input reflection coefficient of normal patch mode.

\section{B. The distance between shorting posts and the patch $L 8$}

Fig. 7 (a) and (b) show the effects of $L 8$ on the resonant frequencies of the monopolar patch and normal patch modes, respectively. As seen from Fig. 7, the resonant frequency of the monopolar patch mode increases when $L 8$ is reduced. However, the resonant frequency of the normal patch mode decreases with $L 8$. This can be attributed to the fact that when $L 8$ decreases, the parasitic capacitance between the shorting posts and the patch $C_{2}$ increases. The increased capacitance $C_{2}$ reduces the inductive effect $\left(L_{i n}\right)$ from the reference plane in Fig. 5 (b), but increases the capacitive effect $\left(C_{i n}\right)$ from the reference plane in Fig. 5 (a) and the $C_{3}$ in Fig. 5 (b). The decreased $L_{\text {in }}$ and the increased $C_{3}$ have reverse effects on the resonant frequency of the monopolar patch mode (the resonant frequency of a parallel $R L C$ circuit). However, the simulation results show that the effect of $L_{i n}$ outweighs that of $C_{3}$, which leads to an increase of the resonant frequency of the monopolar patch mode. On the other hand, the larger capacitance $C_{i n}$ will decrease the resonant frequency of the parallel circuit in Fig. 5 (a), which will shift the resonant frequency of the antenna in normal patch mode to a lower value. 


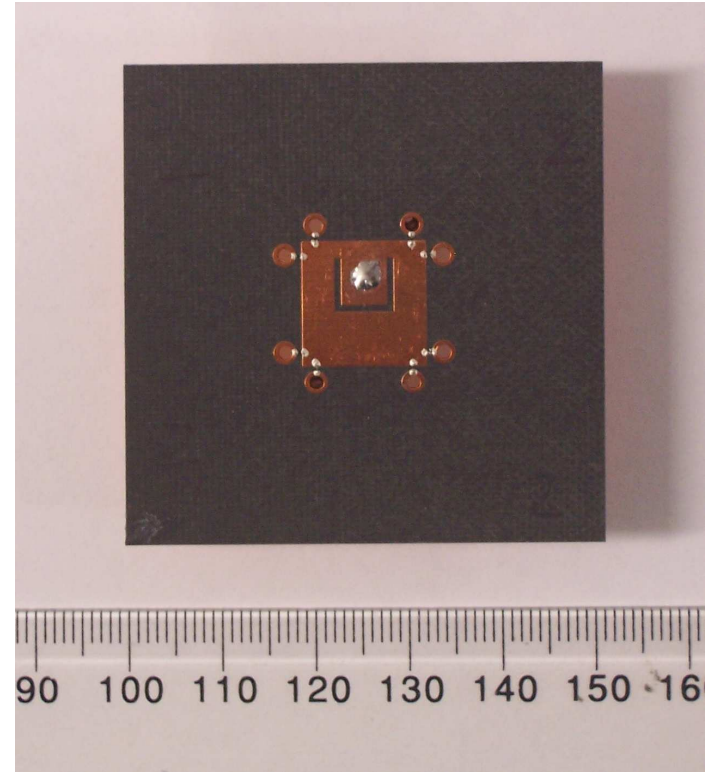

Fig. 8 Photograph of the pattern reconfigurable U-slot antenna.

\section{The number of the shorting posts}

In the design guidelines (Part $A$ of Section II), it is stated that an increase in the number of shorting posts will increase the resonant frequency of the monopolar patch mode, but reduce that of the normal patch mode. The reason is as follows. For the monopolar patch mode, the increased number of shorting posts means that for the diodes in forward bias state, more inductors $\left(L_{\text {in }}\right)$ are paralleled, which makes the effective inductance decrease. And for the diodes in reverse bias state, more capacitors $C_{3}$ are paralleled, which makes the effective capacitance increase. Simulation results show that the decrease of the total inductance outweighs the increase of the total capacitance on the resonant frequency of the $R L C$ circuit in Fig. 5 (b). Therefore, the resonant frequency of the monopolar patch mode is increased when the number of the shorting posts increases. On the other hand, for the normal patch mode, since the PIN diodes connected to the radiating edges are all zero bias, the increased number of shorting posts means more capacitors are paralleled, which makes $C_{i n}$ increase; hence, the resonant frequency of the normal patch mode (the resonant frequency of a parallel $R L C$ circuit in Fig. 5 (a)) will be decreased.

\section{Simulated AND Measured Results of the ANTEnNA}

Based on the above analysis, a pattern reconfigurable U-slot antenna was designed and fabricated. The proposed antenna was etched on a 3.175 -mm-thick RT/Duroid 5880 substrate (dielectric constant $\varepsilon_{r}=2.2, \tan \delta=0.0009$ ). A photograph of the fabricated prototype is shown in Fig. 8. Figs. 9 and 10 show the simulated and measured reflection coefficient versus frequency for three different states of the antenna, respectively. Compared with the simulated results the measured resonant frequencies for State II and III are slightly higher. This discrepancy can be mostly attributed to the inaccuracies in the fabrication of the shorting posts. As is shown in Section III, the

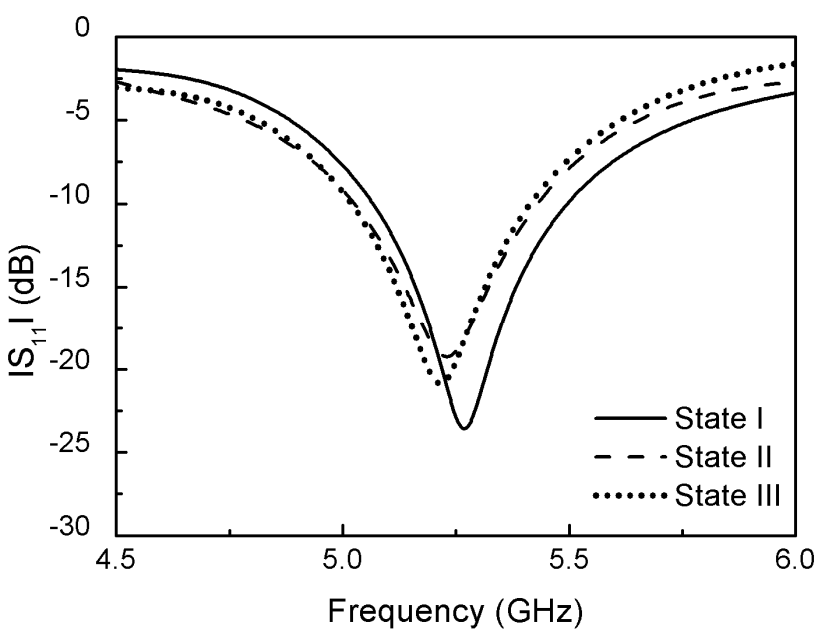

Fig. 9 Simulated input reflection coefficient for the proposed antenna.

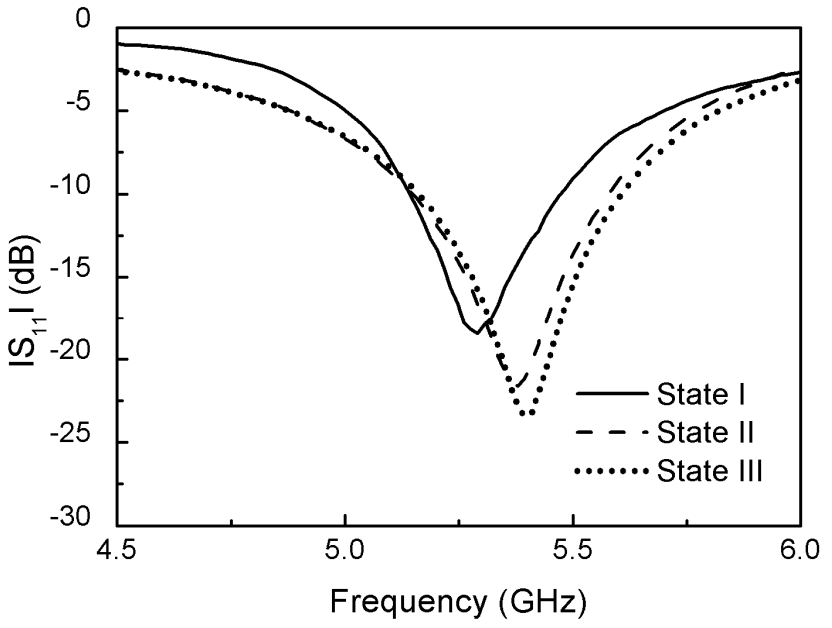

Fig. 10 Measured input reflection coefficient for the proposed antenna.

resonant frequency of the monopolar mode is quite sensitive to the radius and position of the shorting posts. However, the simulated overlapping impedance bandwidth $\left(\left|\mathrm{S}_{11}\right|<-10 \mathrm{~dB}\right)$ of the three states is $6.5 \%$ with a center frequency of $5.24 \mathrm{GHz}$. The corresponding measured bandwidth is $6.6 \%$ centered at $5.32 \mathrm{GHz}$, which agrees reasonably well with the simulated results.

Radiation patterns were measured for the three states of the proposed antenna using a spherical near-field (SNF) antenna measurement system. Simulated and measured normalized radiation patterns are compared for both co-polarization and cross-polarization. Figs. 11 and 12 display the z-x and z-y plane radiation patterns for the three states of the antenna at $5.3 \mathrm{GHz}$, respectively. For State I, boresight radiation patterns with a maximum cross-polarization level of $-20 \mathrm{~dB}$ are shown in Figs. 11 (a) and 12 (a). For State II, a symmetrical conical pattern with the maximum power level in the z-y plane directed at (elevation angle) $44^{\circ}$ is plotted in Figs. 11 (b) and 12 (b). For State III, an asymmetrical conical pattern with the maximum power level in the z-x plane directed at (elevation angle) $45^{\circ}$ is drawn in Figs. 11 (c) and 12 (c). It can be seen from Fig. 11 (c) that the pattern is asymmetrical and there is $1 \mathrm{~dB}$ difference between the left and right maximum power level of the conical 


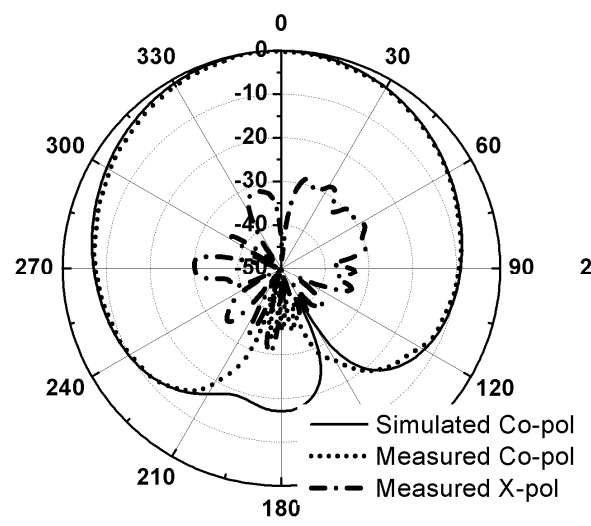

(a)

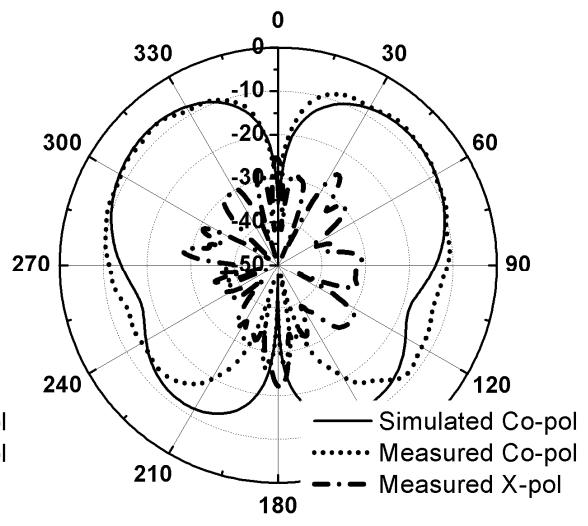

(b)

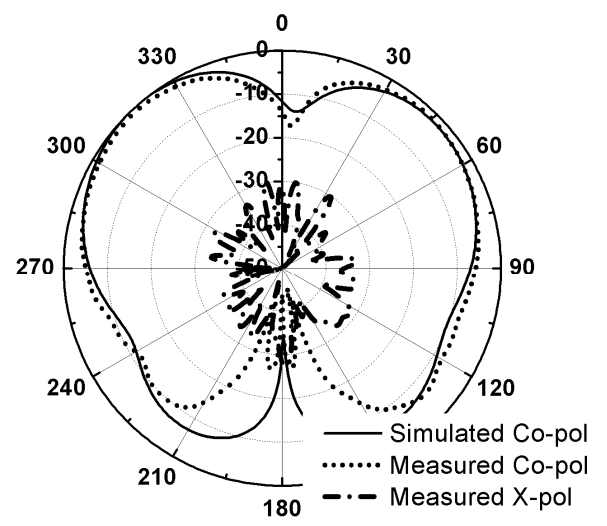

(c)

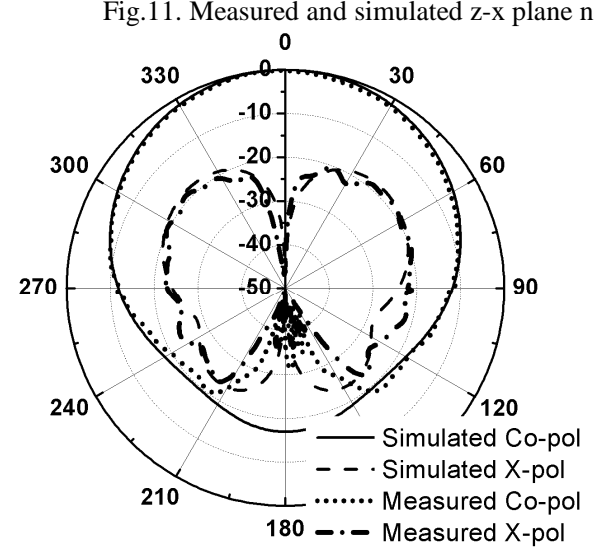

(a)

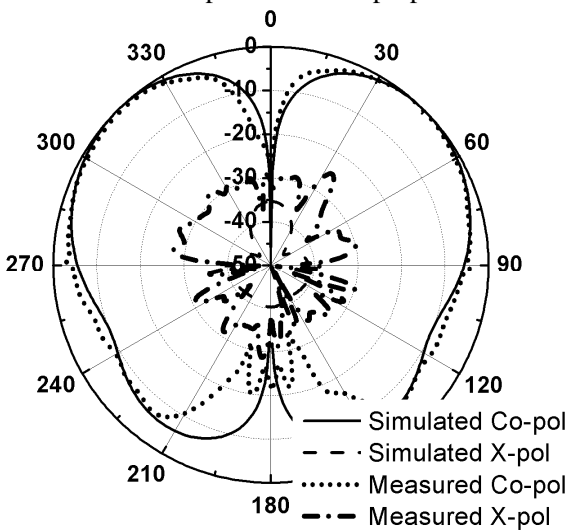

(b)

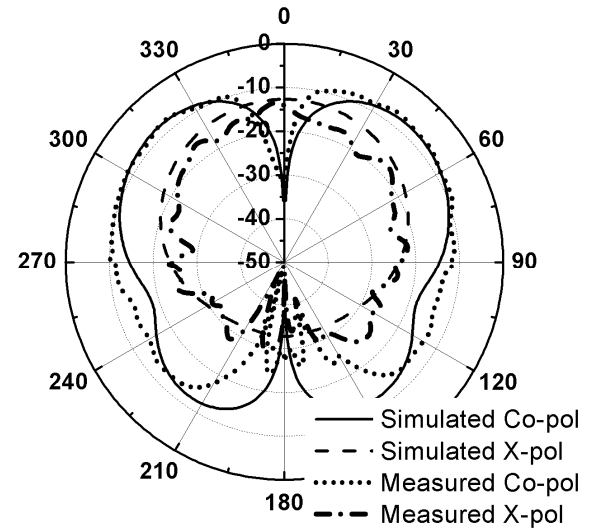

(c)

Fig.12. Measured and simulated z-y plane normalized radiation patterns of the proposed antenna at 5.3 GHz (a) State I (b) State II (c) State III.

pattern. This is due to the position of the probe feed. Simulation results show that if we put the probe feed at the center of the patch, the difference between the left and right maximum power level in Fig. 11 (c) will become smaller. However, in that case the overlapping impedance bandwidth of the two modes will be reduced. This can be viewed as a compromise for the antenna to provide good overlapping impedance bandwidth and radiation patterns. In Fig. 11, the simulated cross-polarization patterns for the three states are not given since they are very small compared to the measured ones.

Additionally, the realized gain was measured using the gain comparison technique [31]. The losses of the cable and bias tee have been calibrated out of the gain measurement. The measured gains for the three states are plotted in Fig. 13. The measured efficiencies of State I, State II and State III at $5.3 \mathrm{GHz}$ are $86.6 \%, 45.1 \%$ and $45.4 \%$, respectively, where the measured efficiency is obtained from the difference between the measured gain and directivity. From Fig. 13, it can be seen that the measured gain of State III is $0.5 \mathrm{~dB}$ greater than that of State II, which can be mostly attributed to the asymmetry of the conical pattern of State III and slight variations in the resistance of individual diodes. Furthermore, at $5.3 \mathrm{GHz}$ the gain of State I is approximately $3 \mathrm{~dB}$ greater than those of State II and III, which is mainly due to the loss of the PIN diodes. On the one hand, for State II and III, four PIN diodes, each acting as a $4 \Omega$ resistor $\left(R_{S}\right)$, are attached to the antenna, but for State I all PIN diodes

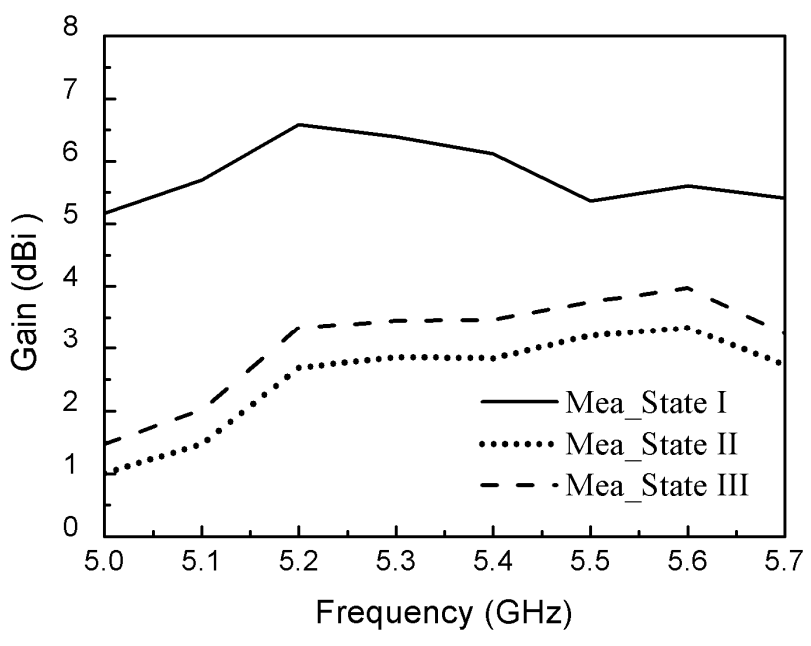

Fig. 13 Measured gain of the proposed antenna.

are turned off. To examine the effect of $R_{S}$, we have simulated several different values of $R_{S}$ at $5.3 \mathrm{GHz}$. Simulation results show that when $R_{S}$ decreases to zero the realized gain increases by $2 \mathrm{~dB}$ and $1.75 \mathrm{~dB}$ for State II and III, respectively. On the other hand, for State II and III, diodes with finite value $R_{P}$ also have more losses than the diodes in zero bias state which is approximately lossless. Simulation results show that at $5.3 \mathrm{GHz}$ when $R_{P}$ changes from $10 \mathrm{k} \Omega$ to infinity the realized gain increases by $0.32 \mathrm{~dB}$ and $0.27 \mathrm{~dB}$ for State II and III, respectively. Therefore, the loss of State II and III is much 


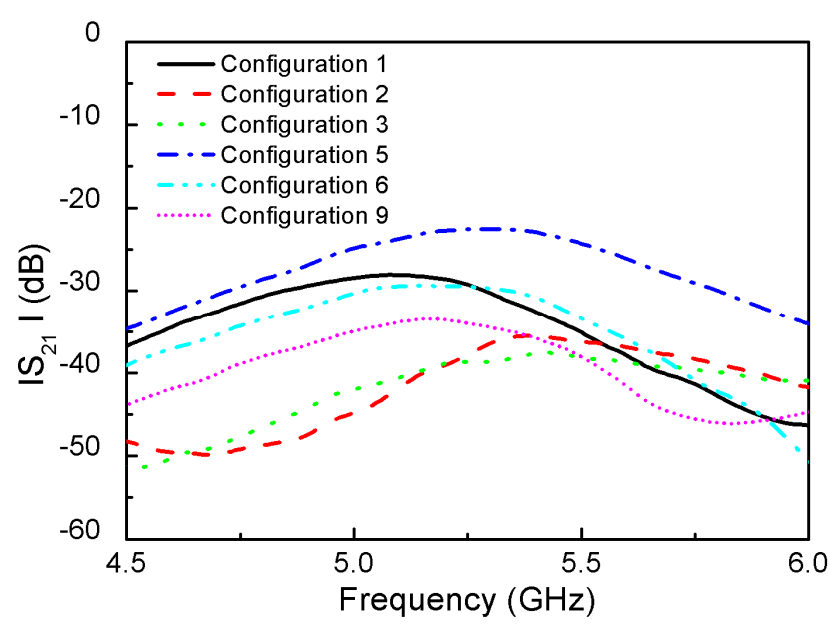

Fig. 14 Measured mutual coupling coefficient for different antenna configurations.

Table III Nine configurations of the reconfigurable antennas at transmit end

\begin{tabular}{|c|c|c|}
\hline Configuration & Antenna 1 & Antenna 2 \\
\hline 1 & State I & State I \\
\hline 2 & State I & State II \\
\hline 3 & State I & State III \\
\hline 4 & State II & State I \\
\hline 5 & State II & State II \\
\hline 6 & State II & State III \\
\hline 7 & State III & State I \\
\hline 8 & State III & State II \\
\hline 9 & State III & State III \\
\hline
\end{tabular}

greater than that of State I and the corresponding gain is much lower. In order to increase the gains of State II and III, low loss elements such as radio frequency microelectromechanical system (RF MEMS) switches could be used. However, the disadvantages of using currently available RF MEMS are the higher cost and lower reliability than PIN diodes.

\section{APPLICATION TO MIMO-OFDM SYSTEMS}

Since the proposed pattern reconfigurable antenna is compact and can vary its radiation pattern without dc bias network, it can be easily applied to a MIMO system. The performance of a MIMO system is affected by the spatial correlation which is a function of the channel characteristics and the antenna array properties. Therefore, the antenna elements can be treated as additional parameters of the MIMO system. In this section, a typical example for the application of pattern reconfigurable antenna in MIMO-OFDM system is described.

\section{A. Antenna array in MIMO-OFDM system}

In previous sections, the design of a single pattern reconfigurable antenna is presented. In this sub-section, we incorporate the pattern reconfigurable antennas into a $2 \times 2$

MIMO-OFDM system.

At each end of the MIMO-OFDM system, there are two reconfigurable antennas working as a two-element array. However, this does not imply that the array operates as a
Table IV Envelope correlation coefficient of nine configurations of the array

\begin{tabular}{|c|c|}
\hline Configuration & $\rho_{\mathrm{e}}$ \\
\hline 1 & $1.12 * 10^{-2}$ \\
\hline 2 & $0.02 * 10^{-2}$ \\
\hline 3 & $0.16 * 10^{-2}$ \\
\hline 4 & $0.02 * 10^{-2}$ \\
\hline 5 & $1.60 * 10^{-2}$ \\
\hline 6 & $0.05 * 10^{-2}$ \\
\hline 7 & $0.16^{*} 10^{-2}$ \\
\hline 8 & $0.05 * 10^{-2}$ \\
\hline 9 & $4.12 * 10^{-2}$ \\
\hline
\end{tabular}

Table V Envelope correlation coefficient of three states of the antenna

\begin{tabular}{|c|c|c|c|}
\hline & State I & State II & State III \\
\hline State I & 1 & 0.013 & 0.07 \\
\hline State II & 0.013 & 1 & 0.33 \\
\hline State III & 0.07 & 0.33 & 1 \\
\hline
\end{tabular}

traditional beamforming array. Rather, each antenna has its own radiation characteristics. As each reconfigurable antenna has three states, there are nine configurations of the antennas at each end. For each measurement, the configuration of the receive antennas is the same as the transmit antennas. Table III gives nine configurations of the reconfigurable antennas.

When two antennas are located close to each other, the mutual coupling can produce pattern distortion and should be eliminated. Therefore, the spacing of the two antennas at both ends is set to be one wavelength in order to keep the mutual coupling to an acceptable level. The measured results in Fig. 14 show that the mutual coupling is lower than $-20 \mathrm{~dB}$ for all configurations at this spacing. The mutual coupling results for configurations 4, 7 and 8 are not shown since they have the same results as the configurations 2,3 and 6 , respectively.

For the reference antenna, in our study, commercially available omnidirectional antennas (Sky-Cross SMA-5250-UA) are used that have omnidirectional patterns with peak gain at 2.2 $\mathrm{dBi}$. The spacing of the two antennas is also set to be one wavelength to keep the mutual coupling below $-20 \mathrm{~dB}$.

When evaluating the MIMO antenna array performance, the envelope correlation coefficient is another critical parameter as it provides a measure of antenna diversity performance. The antenna diversity will be better if the correlation coefficient is lower. The envelope correlation coefficient $\rho_{e}$ can be calculated using the farfield radiation patterns of the antennas [32]

$$
\rho_{e}=\frac{\left|\iint_{4 \pi} \vec{F}_{1}(\theta, \phi) \cdot \vec{F}_{2}^{*}(\theta, \phi) d \Omega\right|^{2}}{\iint_{4 \pi}\left|\vec{F}_{1}(\theta, \phi)\right|^{2} d \Omega \cdot \iint_{4 \pi}\left|\vec{F}_{2}(\theta, \phi)\right|^{2} d \Omega}
$$

where $\vec{F}_{i}(\theta, \phi)$ is a complex vector indicating the electric field radiated from the $i^{\text {th }}$ element. The envelop correlation 


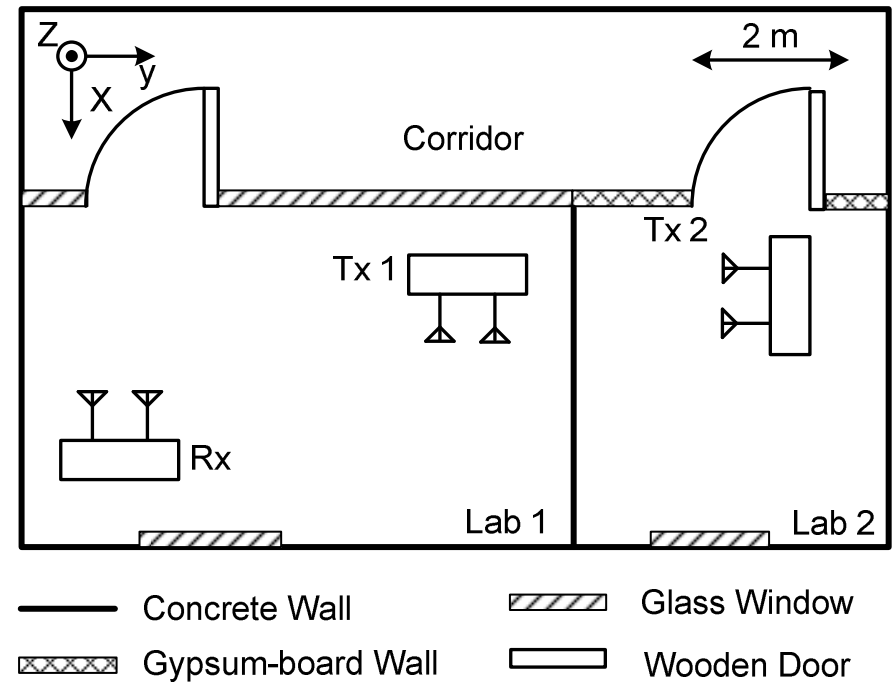

(a)

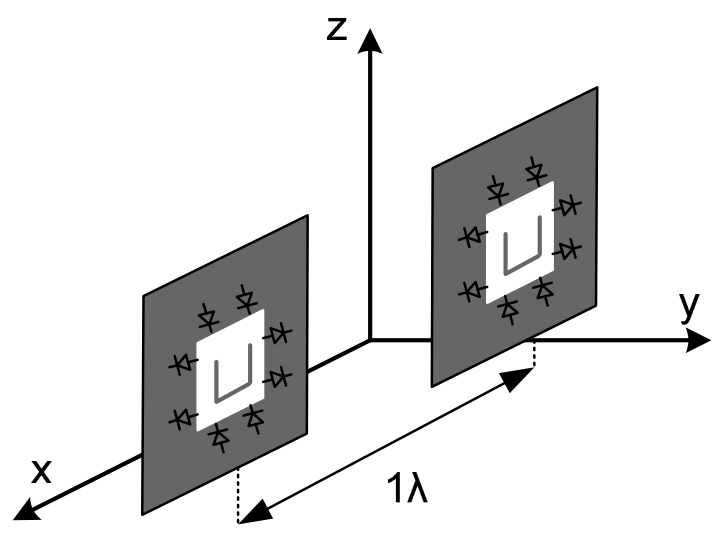

(b)

Fig. 15 (a) Layout of the indoor MIMO-OFDM testing environments; (b) Orientation of the reconfigurable antennas;

coefficient between the patterns generated at the two ports of the antenna array for different configurations (Table III), as well as between the patterns excited by a single antenna for different states (Table II), is estimated by using equation (1) according to the simulated radiation patterns. Table IV gives the envelope correlation coefficient for different antenna configurations at $5.25 \mathrm{GHz}$. It is evident that the envelope correlation coefficients of the configurations $(2,3,4,6,7,8)$ having pattern diversity are lower than those $(1,5,9)$ with the same antenna patterns, and they are comparable to the results of the antennas in [33] that employ both the pattern and polarization diversity. Table $\mathrm{V}$ shows the envelope correlation coefficient for different antenna states at $5.25 \mathrm{GHz}$. It is observed that all the coefficients between different antenna states are below 0.5 , which satisfy the criterion for enabling the antenna to provide a good level of diversity [32].

\section{B. MIMO-OFDM demonstrator}

Actual MIMO-OFDM channel coefficients were measured by the MIMO-OFDM hardware demonstrator developed by CSIRO ICT Centre (Sydney, Australia) [34]. It operates at 5.25 $\mathrm{GHz}$ and supports an operational bandwidth of $40 \mathrm{MHz}$. The

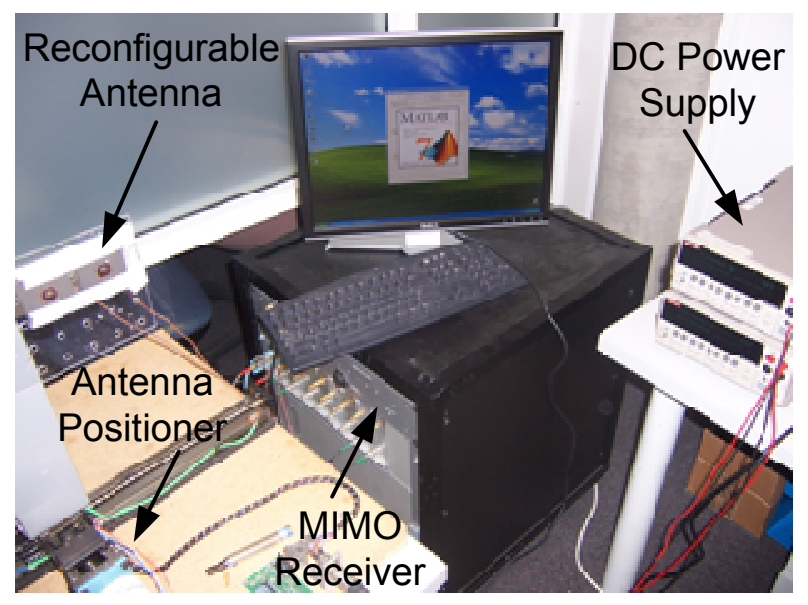

Fig. 16 MIMO-OFDM demonstrator with reconfigurable antennas.

receive antennas are connected to an antenna array positioner controlled by a computer. The channel training sequence is designed to estimate the frequency response over 117 OFDM subcarriers in a $40 \mathrm{MHz}$ bandwidth with a subcarrier spacing of $312.5 \mathrm{kHz}$.

\section{Measurement location and process}

The $2 \times 2$ MIMO-OFDM channel measurement was conducted in the CSIRO ICT Centre indoor environment, which consists of both concrete and gypsum-board walls, glass windows and wooden doors. The channel was measured in LOS over a $5 \mathrm{~m}$ distance and NLOS over an $8 \mathrm{~m}$ distance. The layout of the LOS and NLOS testing scenarios are shown in Fig. 15 (a). For the LOS scenario, both the transmitter and receiver are located in the same laboratory (Lab 1) equipped with some metal bookshelves and cabinets. For the NLOS scenario, the transmitter and receiver are placed in two adjacent laboratories (Lab 1 \& 2).

Each reconfigurable antenna is placed vertically as shown in Fig. 15 (b). In the LOS scenario, the reconfigurable antennas at transmit (Tx1) and receive ends are configured to face each other. In the NLOS scenario, the reconfigurable antennas at transmit (Tx2) and receive ends are configured to face the negative $y$ and negative $x$ directions, respectively. The reference antennas are located with the omnidirectional pattern aligning with the horizontal plane (x-y plane).

For each measurement, the antenna positioner moves the receive antenna array to 10 location samples with 0.05 wavelength increments. The position of the transmit antennas is fixed.

In each scenario only the antenna configuration is changed. Any other factors that can possibly vary the channel characteristics, such as the number of the scatterers and their positions, remain the same. The measurement is performed in the night to avoid the human activities, so the testing environment is entirely static. A photograph of the MIMO-OFDM demonstrator with reconfigurable antennas at the receive end and the dc power supply is shown in Fig. 16. 


\section{Channel measurement and capacity estimation}

The MIMO-OFDM channel is characterized by its coefficient matrix $H(i, j, k, l)$, the element of which is the complex ratio of the signal output from the $i$ th receive antenna over the signal input to the $j$ th transmit antenna at the $k$ th OFDM subcarrier and the $l$ th location sample. The Shannon capacity of a MIMO-OFDM channel is given by [34]

$$
\begin{aligned}
C= & \frac{1}{m \cdot n} \sum_{l=1}^{n} \sum_{k=1}^{m} \log _{2} \\
& \times\left[\operatorname{det}\left(I_{N r}+\frac{S N R}{N_{t}} \overline{H(i, j, k, l)} \cdot \overline{H(i, j, k, l)}^{+}\right)\right]
\end{aligned}
$$

where $C$ is the system capacity in bits/second/Hz, $m$ is the number of sub-carriers, $\mathrm{n}$ is the number of location samples, $N_{r}$ is the number of receive antennas, $N_{t}$ is the number of transmit antennas, $I_{N r}$ is the $N_{r} \times N_{r}$ identity matrix, $\overline{H(i, j, k, l)}$ is the normalized channel matrix, the superscript + denotes the conjugate transpose, and SNR is the average signal-to-noise-ratio over all receive array elements. The capacity of a wideband channel is the average value of the capacities over all subcarriers of the MIMO-OFDM system and the 10 location samples.

It is convenient to use the normalized channel matrix so that the capacity of the channel can be derived as a function of the average SNR per receive antenna over all OFDM subcarriers, receive antenna location samples and MIMO sub-channels. The normalization is performed as follows:

$$
\overline{H(i, j, k, l)}=\frac{H(i, j, k, l)}{\sqrt{\frac{1}{N_{r} \cdot N_{t} \cdot m \cdot n}\|H(i, j, k, l)\|^{2}}}
$$

where $\|H\|$ denotes the Frobenius norm of the channel matrix.

In the MIMO-OFDM channel measurement, the peak gain of the omnidirectional antenna is $2.2 \mathrm{dBi}$. But the gain of different states of the reconfigurable antennas varies from 2.8-6.4 dBi at $5.25 \mathrm{GHz}$. The antenna gain of each configuration is preserved in $\|H\|$. The lower gain of the omnidirectional antenna can lead to a lower value of $\|H\|$. Therefore, compared to the proposed antenna, if all channel matrices are normalized to a common factor, the reference antenna will suffer a capacity loss due to the smaller $\|H\|$.

In this section, we aim to show that the proposed antenna has the ability to improve the system capacity by using pattern diversity to reduce the sub-channel correlation. If the gain of the antenna is taken into account, the extent of the enhancement of the system capacity derived only from the pattern diversity will not be explicitly shown. Therefore, the gain effect on the capacity should be eliminated in order to realize a fair comparison. In order to separate the effect of pattern diversity on the system capacity from the effect of antenna gain, two different channel matrix normalization methods are employed.

The first is that the channel matrix of each antenna configuration is normalized independently (Method I). In this case, the antenna gain is not included in the capacity calculation. Therefore, only the effect of the sub-channel correlation is kept in the capacity evaluation [35]. The second is that the channel

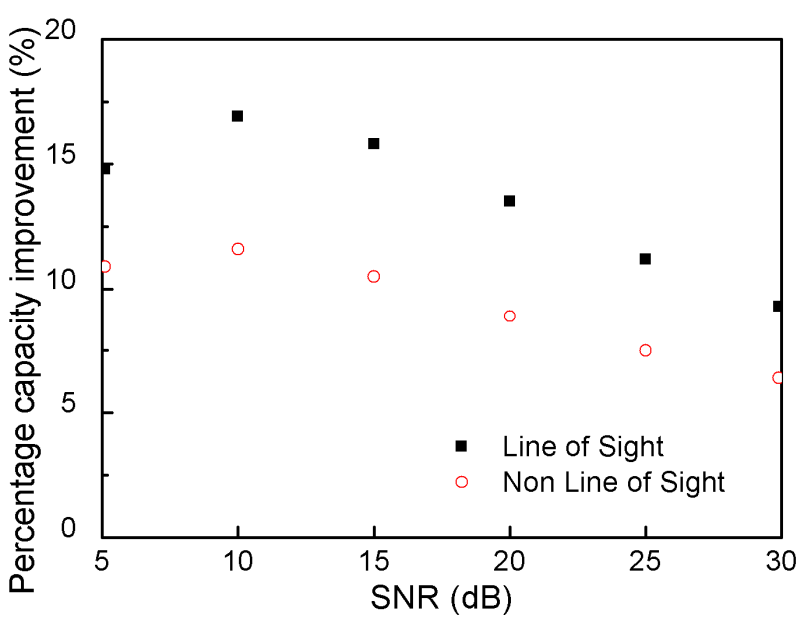

(a)

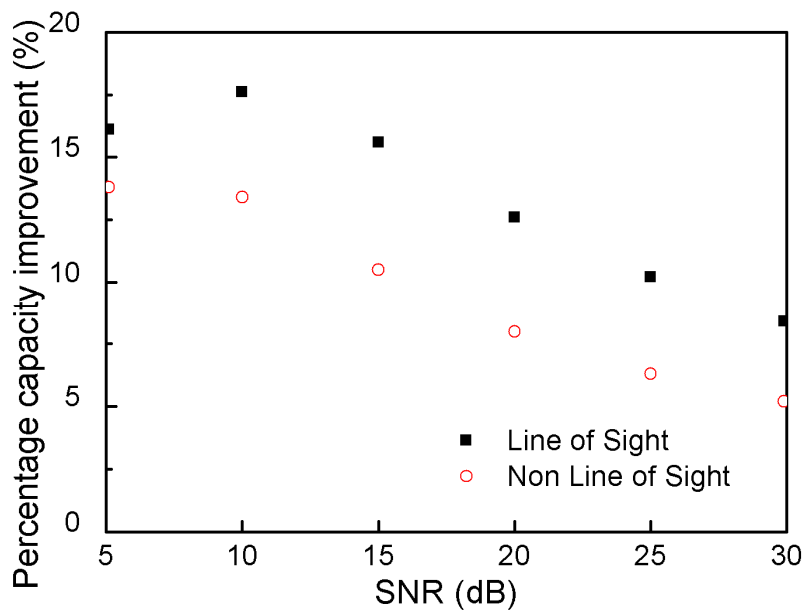

(b)

Fig. 17 Percentage improvement of the reconfigurable antenna MIMO system capacity versus SNR for Method I. The benchmark is (a) omnidirectional antenna and (b) antenna configuration 1.

matrix of each antenna configuration is normalized with respect to that of the reference antenna (Method II). In this way, not only the sub-channel correlation but also the relative received power difference, is preserved in the capacity calculation.

Figs. 17 and 18 show the system capacity percentage improvement for different antenna configurations based on channel normalization Method I and Method II, respectively. For each scenario, the capacity improvement is the difference in capacity between the system with one configuration of reconfigurable antennas which gives the largest capacity and the system with reference antennas. Then the improvement is normalized with respect to the capacity of reference antennas.

For channel matrix normalization Method I, it can be observed from Fig. 17 (a) that compared with omnidirectional antennas, the proposed pattern reconfigurable antennas improve the system capacity by $17 \%$ and $12 \%$ for the LOS and NLOS cases, respectively, at an SNR of $10 \mathrm{~dB}$. This is because that with antenna pattern diversity the correlation of sub-channels is reduced. Therefore, the system capacity is improved, especially for the environments with insufficient scatters. In our experiments the best configurations, which lead to the largest 
system capacity improvement, are the configuration six and eight for the LOS and NLOS scenarios, respectively. An explanation of this is as follows. Although the antennas with omnidirectional patterns in the horizontal plane are considered to be a good solution for MIMO systems as they can receive rich multipath in that plane, it turns out that there are still many multipath components outside the azimuth plane which can be received by other states of the reconfigurable antennas. Therefore, system capacity improvement is possible by using pattern reconfigurable antennas.

In addition, since antenna configuration 1 (Table III) acts as a two-element rectangular patch antenna array with boresight radiation of each element, a comparison of the capacity of the system with configuration 1 and the best configurations is conducted, which is given in Fig. 17 (b). It can be seen that at an SNR of $10 \mathrm{~dB}$, the system capacity is improved by $18 \%$ and $13 \%$ for the LOS and NLOS scenarios, respectively.

Furthermore, as is seen from Fig. 17, the percentage improvement of the system capacity for the LOS scenario is greater than that for the NLOS scenario. This is due to the fact that generally the sub-channel correlation in an indoor LOS environment is larger than that in an NLOS environment. Therefore the extent of improvement of the system capacity by reducing the correlation of MIMO sub-channels is larger for the LOS scenario.

For channel matrix normalization Method II, the best configuration is antenna configuration one (Table III) in terms of the system capacity for both scenarios. It can be seen from Fig. 18 that at an SNR of $10 \mathrm{~dB}$, the peak improvement is $285 \%$ and $264 \%$ for the LOS and NLOS scenarios, respectively, which are much higher than those in Fig. 17. The reason that antenna configuration one outperforms other configurations as well as omnidirectional antennas to such a great extent lies in the higher gain of the antennas in configuration one. Specifically, the $\|H\|$ of the system with configuration one is much greater than that with other configurations. By Method II, this effect is taken into account because the channel matrix of each antenna configuration is normalized to a common value. In this way, the normalized channel matrix of the antenna configuration one is scaled up by a large factor due to the normalization; hence, the improvement of the capacity is significantly larger.

From channel matrix normalization Method I, it is known that the pattern diversity of the proposed antenna can be exploited to improve the system capacity by reducing the sub-channel correlation, which is evidenced by the fact that antenna configurations six and eight outperform the other configurations and the omnidirectional one in the LOS and NLOS scenarios, respectively. When the gain of different configurations is considered, however, it is found that the effect of antenna gain outweighs the effect of the channel decorrelation on the system capacity. Therefore, the antenna configuration one which has the largest antenna gain becomes the best in both scenarios. In this situation, the pattern diversity loses its effect on system

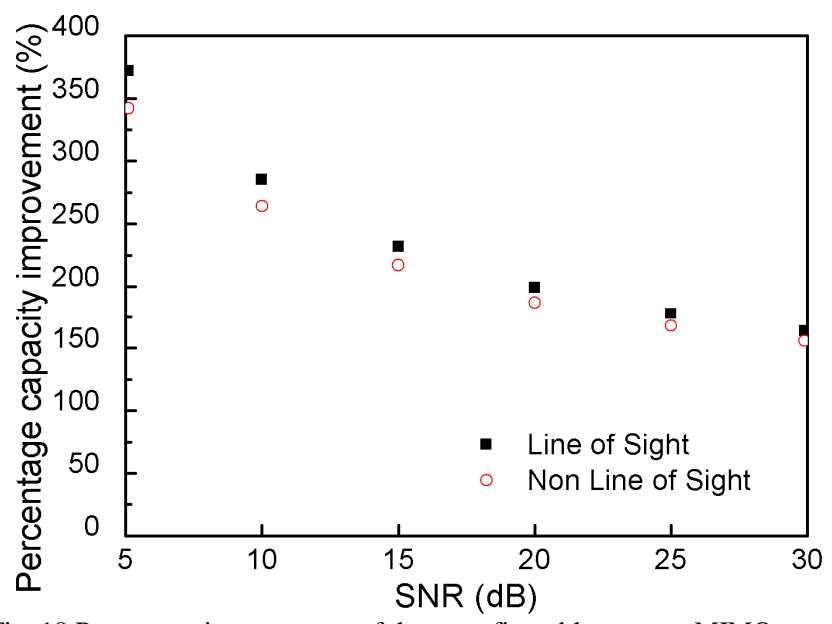

Fig. 18 Percentage improvement of the reconfigurable antenna MIMO system capacity versus SNR for Method II. The corresponding omnidirectional antenna MIMO system capacity is used as the benchmark.

capacity due to the large gain difference of different antenna states.

In order to employ the pattern diversity of the proposed antenna in a practical way that the gain difference is included in the capacity calculation, we need to improve the realized gain of antenna States II and III. As discussed at the end of the Section IV, compared to State I, the lower gain of State II and III is mostly attributed to the losses of PIN diodes. Therefore, low loss switches, such as RF MEMS, should be used to make the gain of States II and III close to that of State I. An updated antenna prototype with RF MEMS could be designed and adopted in our future work.

Reference [17] also demonstrates the capability of a pattern reconfigurable antenna to improve the MIMO system capacity. Compared to [17], our work has three main differences. First, the mechanism of the pattern reconfigurability of the antenna in [17] uses mutual coupling of two closely spaced antennas. The pattern diversity will be reduced if the antenna separation is increased. Therefore, the spatial diversity, which is another effective scheme [36] to improve the system capacity, can not be efficiently employed together with the pattern diversity in a MIMO system. However, as the way to reconfigure the pattern of our antenna is changing the antenna operating mode, there is no space limitation on our antenna. Second, in our experiments, at an SNR of $10 \mathrm{~dB}$, the capacity percentage improvement is $17 \%$ and $12 \%$ for the LOS and NLOS scenarios, respectively. These results are larger compared to the average 10\% improvement reported in [17]. Admittedly, since the capacity measurement is heavily dependent on the testing environment, the improvement could be significantly different for other test scenarios. Third, as has already been mentioned in the introduction, the reference antenna in [17] is chose to be one configuration of the antenna. While in our work, omnidirectional antennas are used for reference. Since omnidirectional antennas can receive rich multipath in the horizontal plane, this comparison can highlight the effect of pattern diversity on MIMO system capacity. 


\section{CONCLUSION}

A new design of a pattern reconfigurable patch antenna is presented. Shorting posts around the patch are used to change the antenna operating modes. The antenna can switch between three different radiation patterns by employing PIN diodes. It is compact but can realize an overlapping frequency bandwidth of $6.6 \%$ with a center frequency of $5.32 \mathrm{GHz}$ for the three states by etching a U-slot into the patch. Compared to most conventional pattern reconfigurable antennas, the proposed antenna does not need additional dc bias lines to control the PIN diodes, which greatly simplifies the antenna structure. Due to the simple structure and pattern reconfigurability, the antenna has the ability to improve the performance of a wireless communication system considerably.

To demonstrate the antenna's capability of increasing the system capacity, four antennas are applied to a $2 \times 2$ MIMO-OFDM system and the indoor channel measurement is conducted. Capacities of the system with pattern reconfigurable antennas and reference omnidirectional antennas are compared based on two channel matrix normalization methods. The comparison indicates that, for normalization Method I, the system capacity is improved by $17 \%$ and $12 \%$ for the LOS and NLOS scenarios, respectively, at an SNR of $10 \mathrm{~dB}$ by using the pattern reconfigurable antennas. When the gain effect is considered, the configuration with the greatest antenna gain produces the largest system capacity. Therefore, in order to use the pattern diversity to enhance the system capacity effectively in a practical way, the gain of antenna States II and III needs to be improved to approach that of State I.

Future research includes integrating RF MEMS switches with the proposed antenna to increase the gain of antenna States II and III. Furthermore, work will focus on designing combined pattern and polarization reconfigurable antennas to achieve higher system capacity improvement.

\section{ACKNOWLEDGMENT}

The authors would like to thank Mei Shen for assisting with the attachment of PIN diodes and Dr. Hajime Suzuki, Dr. Zhuo Chen and Dr. Wei Ni for the discussion on the subject of MIMO communication. In addition, we appreciate the constructive comments from the anonymous reviewers.

\section{REFERENCES}

[1] G. H. Huff, J. Feng, S. Zhang, and J. T. Bernhard, "A novel radiation pattern and frequency reconfigurable single turn square spiral microstrip antenna," IEEE Microw. Wireless Compon. Lett., vol. 13, pp. 57-59, Feb. 2003.

[2] A. R. Weily, T. S. Bird, and Y. J. Guo, "A reconfigurable high-gain partially reflecting surface antenna," IEEE Trans. Antennas Propag., vol. 56, no. 11, pp. 3382-3390. Nov. 2008.

[3] P. Y. Qin, A. R. Weily, Y. J. Guo, T. S. Bird, and C. H. Liang, "Frequency reconfigurable quasi-yagi folded dipole antenna," IEEE Trans. Antennas Propag., vol. 58, no. 8, pp. 2742-2747. Aug. 2010.

[4] S. V. Hum and H. Y. Xiong, "Analysis and Design of a Differentially-Fed Frequency Agile Microstrip Patch Antenna," IEEE Trans. Antennas Propag., vol. 58, no. 10, pp. 3122-3130. Oct. 2010.
[5] P. Y. Qin, A. R. Weily, Y. J. Guo, and C. H. Liang, "Polarization reconfigurable u-slot patch antenna," IEEE Trans. Antennas Propag., vol. 58, No.10, pp. 3383-3388, Oct. 2010.

[6] F. Yang and Y. Rahmat-Samii, "A reconfigurable patch antenna using switchable slots for circular polarization diversity," IEEE Microw. Wireless Compon. Lett., vol. 12, no. 3, pp. 96-98, Mar. 2002.

[7] S. Zhang, G. H. Huff, J. Feng, and J. T. Bernhard, "A Pattern reconfigurable microstrip parasitic array," IEEE Trans. Antennas Propag., vol. 52, no. 10, pp. 2773-2776, Oct. 2009.

[8] S. L. S. Yang and K. M. Luk, "Design a wide-band L-probe patch antenna for pattern reconfigurable or diversity applications," IEEE Trans. Antennas Propag., vol. 54, no. 2, pp. 433-438, Feb. 2006.

[9] S. H. Chen, J. S. Row, and K. L. Wong, "Reconfigurable square-Ring patch antenna with pattern diversity," IEEE Trans. Antennas Propag., vol. 55, no. 2, pp. 472-475, Feb. 2007.

[10] W. L. Liu, T. R. Chen, S. H. Chen, and J. S. Row, "Reconfigurable microstrip antenna with pattern and polarization diversities," Electron. Lett., vol. 43, no. 2, pp.77-78, Jan. 2007.

[11] M. A. Jensen and J. W. Wallace, "MIMO wireless channel modeling and experimental characterization" in Space-Time Processing for MIMO Communications, A. B. Gershman and N. D. Sidiropoulos, Eds. West Sussex, U.K.: Wiley, 2005.

[12] P. Kyritsi, D. C. Cox, R. A. Valenzuela, and P. W. Wolniansky, "Effect of antenna polarization on the capacity of a multiple element system in an indoor environment," IEEE J. Sel. Areas Commun., vol. 20, no.6, pp.1227-1239, Aug. 2002.

[13] P. Y. Qin, Y. J. Guo, and C. H. Liang, "Effect of antenna polarization diversity on MIMO system capacity," IEEE Antennas Wireless Propag. Lett., vol. 9, pp. 1092-1095. Dec. 2010.

[14] B. A. Cetiner, E. Akay, E. Sengul, and E. Ayanoglu, "A MIMO system with multifunctional reconfigurable antennas," IEEE Antennas Wireless Propa. Lett., vol. 5, pp. 463-466, Dec. 2006.

[15] A. Grau, J. Romeu, M. J. Lee, S. Blanch, L. Jofre and F. D. Flaviis, “ A dual-linearly-polarized MEMS-reconfigurable antenna for narrowband MIMO communication systems," IEEE Trans. Antennas Propag., vol. 58, no. 1, pp. 4-17, Jan. 2010.

[16] J. D. Boerman and J. T. Bernhard, "Performance study of pattern reconfigurable antennas in MIMO communication systems," IEEE Trans. Antennas Propag., vol. 56, no. 1, pp. 231-236, Jan. 2007.

[17] D. Piazza, N. J. Kirsch, A. Forenza, R. W. Heath, Jr., and K. R. Dandekar, "Design and evaluation of a reconfigurable antenna array for MIMO systems," IEEE Trans. Antennas Propag., vol. 56, no. 3, pp. 869-881, Mar. 2008.

[18] P. Mookiah, D. Piazza, and K. R. Dandekar, "Reconfigurable spiral antenna array for pattern diversity in wideband MIMO communication systems," in Proc. IEEE Antennas and Propag. Society Int. Symp., Jul. 2008.

[19] H. K. Pan, et al., "Increasing channel capacity on MIMO system employing adaptive pattern/polarization reconfigurable antenna," in Proc. IEEE Antennas and Propag. Society Int. Symp., Jun. 2007.

[20] D. Piazza, M. D'Amico, and K. R. Dandekar, "Performance improvement of a wideband MIMO system by using two-port RLWA," IEEE Antennas Wireless Propag. Lett., vol. 8, pp. 830-834, 2009.

[21] P. Y. Qin, A. R. Weily, Y. J. Guo, C. H. Liang, and Y. Cai, “A pattern reconfigurable U-slot patch antenna," in Proc. IEEE Antennas and Propag. Society Int. Symp., Jul. 2010.

[22] I. J. Bahl and P. Bhartia, Microstrip Antennas. New York: Artech House, 1980

[23] C. Delaveaud, P. Leveque, and B. Jecko, "New kind of microstrip antenna: the monopolar wire-patch antenna," Electron. Lett., vol. 30, no. 1, pp. 1-2, Jan. 1994.

[24] P. Bhartia and I. J. Bahl, "Frequency agile microstrip antennas," Microwave Journal, pp. 67-70, Oct. 1982.

[25] D. L. Sengupta, "Resonant frequency of a tunable rectangular patch antenna," Electron. Lett., vol. 20, no. 15, pp. 614-615, Jul. 1984.

[26] CST Studio SuiteTM 2009, Computer Simulation Technology, Germany.

[27] T. Huynh and K. F. Lee, "Single-layer single-patch wideband microstrip antenna," Electron. Lett., vol. 31, no. 16, pp. 1310-1312, Aug. 1995.

[28] M/A-COM Data Sheet for MA4AGBLP912 Beam lead PIN Diode.

[29] S. Weigand, G. H. Huff, K. H. Pan, and J. T. Bernhard, "Analysis and design of broad-band single-layer rectangular U-slot microstrip patch 
antennas," IEEE Trans. Antennas Propag., vol. 51, no. 3, pp. 457-468, Mar. 2003.

[30] J. Anguera, E. Martinez, C. Puente, C. Borja, and J. Soler, "Broad-band dual-frequency microstrip patch antenna with modified sierpinski fractal geometry," IEEE Trans. Antennas Propag., vol. 52, no. 1, pp. 66-72, Jan. 2004.

[31] C. A. Balanis, Antenna Theory: Analysis and Design, 3rd ed. New York: Wiley, 2005.

[32] R. Vaughan and J. Andersen, "Antenna diversity in Mobile Communications," IEEE Trans. Veh. Technol., vol. VT-36, no. 4, pp. 149-172, Nov. 1987.

[33] Z. N. Chen, T. S. P. See, "Diversity and its applications in ultra-wideband antennas," IEEE Int. Workshop on Antenna Tech., Santa Monica, California, Mar. 2009.

[34] H. Suzuki, T. V. Tran, and I. B. Collings, "Characteristics of MIMO-OFDM Channels in indoor environments," J. Wireless Commun. Netw., vol. 2007, no.19728, Jan., 2007.

[35] M. A. Jensen and J. W. Wallace, "A review of antennas and propagation for MIMO wireless communications," IEEE Trans. Antennas Propag., vol. 52, no. 11, pp. 2810-2824, Nov. 2004.

[36] C. B. Dietrich, Jr., K. Dietze, J. R. Nealy, and W. L. Stutzman, "Spatial, polarization, and pattern diversity for wireless handheld terminals," IEEE Trans. Antennas Propag., vol. 49, no. 9, pp. 1271-1281, Sep. 2001. 